\title{
A DYNAMICAL SYSTEMS MODEL OF THE LIMITING OXYGEN INDEX TEST
}

\author{
M. I. NELSON ${ }^{1}$, H. S. SIDHU ${ }^{2}$, R. O. WEBER ${ }^{2}$ and G. N. MERCER ${ }^{2}$
}

(Received November 29, 1998; revised February 2, 1999)

\begin{abstract}
Oxygen index methods have been widely used to measure the flammability of polymeric materials and to investigate the effectiveness of fire-retardants. Using a dynamical systems framework we show how a limiting oxygen index can be identified with an appropriate bifurcation.

The effectiveness of fire-retardants in changing the limiting oxygen index is calculated by unfolding the bifurcation point with a suitable non-dimensionalised variable, which depends upon the mode of action of the additive. In order to use this procedure it is essential the model is non-dimensionalised so as to retain the variables of interest as distinct continuation parameters.
\end{abstract}

\section{Introduction}

The burning behaviour of polymeric materials is understood in terms of their ability to generate flammable volatile liquids and gases under the action of heat and their subsequent ignition. The flammability of a given polymer depends upon both the physical state of the product in which it appears and the particular fire scenario that is considered. Accordingly there exists a multitude of standard test methods to assess flammability. Many of these assess the potential hazard around identifiable pass/fail criteria: it is usually not possible to quantify the degree of pass or failure achieved. They can therefore not be used to rank the relative flammability of a selection of products.

Oxygen index methods, which describe the tendency of a material to sustain a flame, have gained considerable acceptance as a tool to investigate the flammability of polymers [5]. One of their strengths is that they provide a convenient, reproducible,

\footnotetext{
'Department of Fuel and Energy, The University of Leeds, Leeds LS2 9JT, England.

${ }^{2}$ School of Mathematics and Statistics, University College, University of New South Wales, Australian Defence Force Academy, Canberra 2600, Australia.

(C) Australian Mathematical Society 2001, Serial-fee code 0334-2700/01
} 


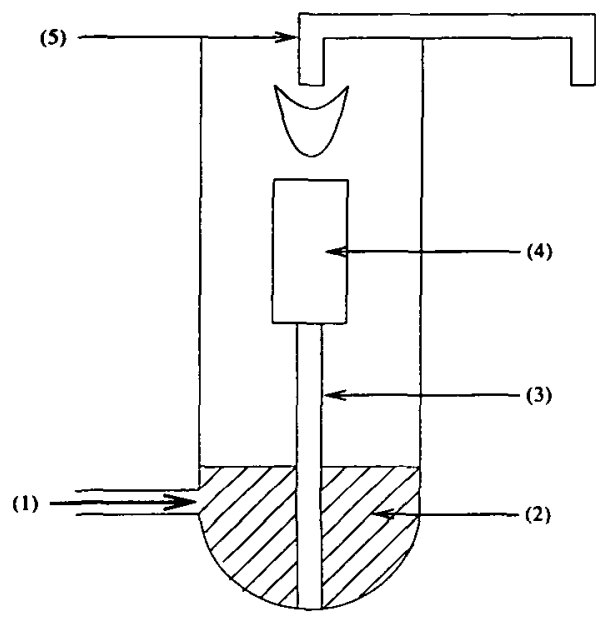

(a) Schematic diagram of apparatus for determination of oxygen index.

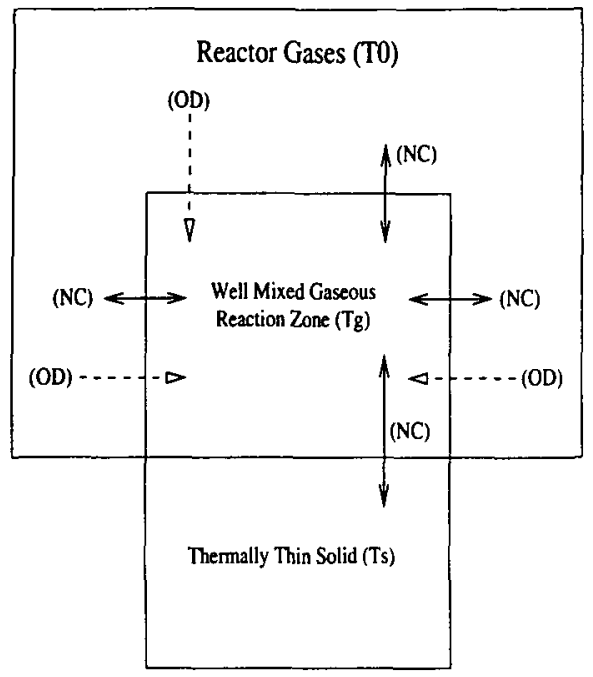

(b) Model geometry.

FIGURE 1. Schematic oxygen index test apparatus and model geometry. (a): (1) Inflow of $\mathrm{N}_{2}-\mathrm{O}_{2}$ mixture. (2) Glass beads. (3) Clamp with rod support. (4) Sample. (5) Ignitor. (b): (NC) Newtonian Cooling. (OD) Flow of oxygen from the reactor gases (at the inflow concentration $\mathscr{O}_{2, a}$ ) into the gaseous reaction zone. (C)2001, The Institute of Physics. Reprinted from [7].

means of determining a numerical measure of flammability. They have therefore been used as the basis for systematic investigations into the relative flammabilities of fireretarded materials and consequently into the effectiveness of different fire-retardants and fire-retardancy mechanisms.

Figure 1 (a) provides a schematic diagram of a typical oxygen-index method. The quintessential feature of the apparatus is that the oxygen concentration in the inflow gases is controllable. The standard experimental procedure is to ignite the top of the sample, using a gas flame, withdrawing the igniter once ignition has occurred, and to find the lowest oxygen concentration at which a "minimum burning length" criterion is met. This criterion typically takes the form of either specifying that the sample must burn for a certain length of time or that a specified length of material be consumed. The limiting oxygen index (LOI), also called the critical oxygen index (COI) or oxygen index $(\mathrm{OI})$, is then defined as:

$$
\mathrm{LOI}=\frac{\phi_{c r}\left(\mathscr{O}_{2}\right)}{\phi_{c r}\left(\mathscr{O}_{2}\right)+\phi\left(\mathscr{N}_{2}\right)},
$$

where $\phi_{c r}\left(\mathscr{\sigma}_{2}\right)$ and $\phi\left(\mathscr{N}_{2}\right)$ are the minimum oxygen concentration in the inflow gases 
required to pass the "minimum burning length" criterion and the nitrogen concentration in the inflow gases respectively.

Assuming that the inflow gases contain only oxygen and nitrogen then $\phi_{c r}\left(\mathscr{O}_{2}\right)+$ $\phi\left(\mathscr{N}_{2}\right)=1$ and $(1)$ becomes

$$
\mathrm{LOI}=\phi_{c r}\left(\mathscr{O}_{2}\right)
$$

The limiting oxygen index is more commonly reported as a percentage rather than as a fraction.

Since air comprises about $20.95 \%$ oxygen by volume, any material with a limiting oxygen index less than this will burn easily in air. Conversely, the burning behaviour and tendency to propagate flame for a polymer with a limiting oxygen index greater than 20.95 will be reduced or even zero after removal of the igniting source. Selfsustaining combustion in any oxygen-nitrogen atmosphere is not possible if LOI > 100 , indeed such values are not physically meaningful.

No classification of burning behaviour based upon the limiting oxygen index has gained wide acceptance. However, several researchers have suggested that "selfextinguishing" polymers have a limiting oxygen index greater than 28 [5]. This is the definition that we use in this paper. In addition we define materials with a limiting oxygen index of 20.95 as being "marginally stable" and those with LOI $>100$ as "intrinsically inflammable"; the latter should be understood to be shorthand for "intrinsically inflammable in the context of a limiting oxygen index test'.

We refer the reader interested in additional details of oxygen-index methods and their applications, particularly to assessing the burning behaviour of textiles, to the extensive review of Horrocks $\mathrm{et}$ al. [5], and references therein.

\subsection{Simplified models for polymer combustion A complete description of the} mechanisms leading to the establishment of a flame over a burning surface requires consideration of mass and heat transport in both the gas and solid phases. Although the overall phenomena are complicated, two salient processes, one in each phase, must occur if a material is to ignite. The solid must first decompose to release volatiles into the boundary layer. These gases must then mix with surrounding air to produce a flammable mixture, which then either autoignites or is ignited by an external source, such as a pilot flame. Traditionally fire scientists have used highly simplified models which, typically, examine these key processes in isolation. Recently nonlinear dynamical systems models have been developed that describe these processes $[1,6,8-10]$.

\subsection{Dynamical systems models for limiting-oxygen index tests Two dynamical} systems models for oxygen-index experiments have been investigated by Rychlý and co-workers $[1,8]$. In these papers the polymer is 'ignited' and the concentration of 
oxygen varied until the flame becomes unstable, that is, its temperature decreases to the temperature of the surrounding medium. Although this is not the definition used in the oxygen-index standards, it is closely related to it: if the flame is stable then all of the sample eventually burns, if it is unstable only a fraction of it burns. In the latter case the proportion of the sample that burns is determined by how quickly the flame goes out; a limiting oxygen index defined directly in terms of flame stability will be at least equal in value to one defined in terms of a "minimum burning length", and usually greater. In addition to its link with the limiting oxygen index, the definition of Rychlý et al. of flame stability is of theoretical interest in its own right.

In the first limiting oxygen index model [1] the concentration of gas-phase species is given in 'relative units'. It is not stated how these are related to SI units. Furthermore, it is unclear how many of the model parameters are defined in terms of standard physical and chemical constants; some physical constants are only defined implicitly. Their second model [8] is a vast improvement in this respect, although there remain aspects which are poorly defined. In both models there are physical and chemical processes that are inconsistently modelled.

Our purposes in this paper are twofold. We first introduce a model, closely following the approach pioneered by Rychlý et al. [1,8-10], in which physical processes are explicitly stated and which does not contain any inconsistencies. Rychlý and coworkers have investigated the properties of their models by direct integration of the governing equations, including consumption of the solid polymer. However, the definition of limiting oxygen index that they use suggests an interpretation in terms of bifurcation theory. Our second purpose therefore is to show how a limiting oxygen index can be defined in a steady-state formulation and subsequently to use continuation methods to investigate its dependence upon parameters of interest, such as the concentration of oxygen in the inflow gases.

\section{Description of the model}

In establishing our model we consider three zones: the solid sample, where pyrolysis occurs, a gaseous reaction zone, where gas-phase reactions occur, and a 'reactor gases' zone. Figure 1 (b) shows the geometry of the model.

2.1. Model physics Pyrolysis of the sample is assumed to occur over a thermally-thin control volume. Heat transfer across the bottom face and sides of this is assumed to be negligible. Heat transfer between the upper face of the sample and the gaseous reaction zone is modelled by a Newtonian cooling term. For mathematical convenience we assume that there are no significant temperature gradients across the control volume, so that it has a uniform temperature. The products of the thermal degradation reaction, (3), are assumed to diffuse into the gaseous reaction zone instantaneously. 
The gaseous reaction zone is assumed to be a well-mixed cuboid. The sides which undergo Newtonian cooling with either the zone of reactor gases or the test material, are as indicated in Figure 1 (b). Fresh oxygen flows into the gaseous reaction zone from the reactor gas zone, where its concentration is equal to that in the inflow gases. The flow of oxygen into the gaseous reaction zone is modelled as a first-order 'reaction' term. The reactor gas zone is assumed to be at a constant temperature $\left(T_{0}\right)$ which reflects the temperature of the heat source used to ignite the sample.

2.2. Model chemistry Combustion chemistry is modelled by a single reaction in the solid-phase and two reactions in the gas-phase. The solid phase reaction represents the thermal degradation of the test material $\left(\mathscr{M}_{1}\right)$ as a single-step reaction (heat of reaction $\left.Q_{s}\right)$. The products of this reaction $\left(\mathscr{M}_{2}\right)$ flow into the gaseous reaction zone where they either undergo oxidation (heat of reaction $Q_{g}$ ) or participate in 'incomplete combustion reactions' (heat of reaction $Q_{i}$ ). The products of these reactions, $\mathscr{M}_{3}$ and $\mathscr{M}_{4}$, are assumed to have no effect on the system dynamics.

The model chemistry is thus given by:

$$
\begin{aligned}
\mathscr{M}_{1} \rightarrow \mathscr{M}_{2} & \text { Reaction } 0 \text { Polymer Degradation: Endothermic; } \\
\mathscr{M}_{2}+\mathscr{O}_{2} \rightarrow \mathscr{M}_{3} & \text { Reaction } 1 \text { Gas Phase Oxidation: Exothermic; } \\
\mathscr{M}_{2} \rightarrow \mathscr{M}_{4} & \text { Reaction } 2 \text { Incomplete Gas Phase Reactions: Endothermic. }
\end{aligned}
$$

\section{Model equations}

3.1. Dimensional equations For a given 'material' we fix thermophysical and chemical properties and treat the concentration of oxygen in the inflow gases, $\mathscr{O}_{2, a}$, the experimentally controlled parameter, as the primary bifurcation parameter. We seek the smallest value of $\mathscr{O}_{2 . a}$, which we call $\mathscr{O}_{2 . a . c r}$, such that a flame is sustained. We are interested in how this critical value depends upon other model parameters.

The system that we study is

$$
\begin{aligned}
V_{g} \rho_{g} c_{p_{s}} \frac{\mathrm{d} T_{g}}{\mathrm{~d} t}= & V_{g} Q_{g} A_{g} e^{-E_{g} / R T_{s}} \mathscr{M}_{2} \mathscr{O}_{2}-V_{g} Q_{i} A_{i} e^{-E_{i} / R T_{s}} \mathscr{M}_{2} \\
& -S_{g . o} \chi_{g . o}\left(T_{g}-T_{0}\right)-S_{g . s} \chi_{g . s}\left(T_{g}-T_{s}\right), \\
V_{s} \rho_{s} c_{p_{s}} \frac{\mathrm{d} T_{s}}{\mathrm{~d} t}= & -Q_{s} A_{s} e^{-E_{s} / R T_{s}} \mathscr{M}_{1}+S_{g . s} X_{g . s}\left(T_{g}-T_{s}\right), \\
V_{g} \frac{\mathrm{d} \mathscr{M}_{2}}{\mathrm{~d} t}= & n A_{s} e^{-E_{s} / R T_{s}} \mathscr{M}_{1}-V_{g} A_{g} e^{-E_{g} / R T_{s}} \mathscr{M}_{2} \mathscr{O}_{2} \\
& -V_{g} A_{i} e^{-E_{i} / R T_{g}} \mathscr{M}_{2}-L \mathscr{M}_{2}, \\
V_{g} \frac{\mathrm{d} \mathscr{O}_{2}}{\mathrm{~d} t}= & -V_{g} A_{g} e^{-E_{z} / R T_{s}} \mathscr{M}_{2} \mathscr{O}_{2}+V_{g} A_{3} e^{-E_{3} / R T_{g}}\left\{\mathscr{O}_{2 . a}-\mathscr{O}_{2}\right\},
\end{aligned}
$$




$$
\begin{aligned}
\frac{\mathrm{d} \mathscr{M}_{1}}{\mathrm{~d} t} & =-A_{s} e^{-E_{s} / R T_{s}} \mathscr{M}_{1}, \\
A_{s} & =\frac{\mathscr{H} E_{s}}{R T_{c}^{2}} e^{E_{s} / R T_{c}}, \\
T_{g}(0) & =T_{s}(0)=T_{\text {ref }}, \\
\mathscr{M}_{1}(0) & =V_{s} \rho_{s}, \quad \mathscr{M}_{2}(0)=0, \quad O_{2}(0)=\mathscr{O}_{2, a} .
\end{aligned}
$$

The terms that appear in equations (6)-(13) are defined in a related paper [7].

We define the pre-exponential factor of the pyrolysis reaction, (11), in terms of a characteristic temperature, which can be measured in a thermogravimetric experiment. This has proved successful in modelling the behaviour of polymeric materials in the cone calorimeter and is explained elsewhere [6]. The characteristic temperature can be considered as a crude measure of the temperature at which a polymer decomposes.

Equation (6) models the temperature of the gaseous reaction zone. The first two terms on the right-hand side of this equation represent gas-phase chemistry, see Section 2.2, and are due to the exothermic gas-phase oxidation and the endothermic incomplete combustion reactions respectively. The last two terms in (6) model heat transfer between the gaseous reaction zone and the reactor gases/the polymer surface respectively.

Equation (7) models the temperature of the polymer. The first term on the righthand side of this equation represents the heat required to gasify the solid and is the product of the rate of gasification, at temperature $T_{s}$, and the heat of gasification, which is endothermic. The second term models heat transfer between the polymer surface and the gaseous reaction zone.

Equation (8) models the concentration of fuel in the gaseous reaction zone. The first term on the right-hand side of this equation represents the flow of pyrolysis products, produced by decomposition of the polymer, into the gaseous reaction zone. The second and third terms denote depletion of the fuel due to an oxidation reaction and incomplete combustion reactions respectively. The fourth term is due to the movement of the inflow gases through the reactor chamber, see Figure 1 (a), which removes fuel from the gaseous reaction zone.

Equation (9) models the concentration of oxygen in the gaseous reaction zone. The first term on the right-hand side of this equation is due to the depletion of oxygen in the oxidation reaction. The second term models the rate of oxygen diffusion into the flame [9].

Equation (10) models the degradation of the polymer. The degradation kinetics are assumed to be Arrhenius and first order with respect to the polymer. To simplify the problem we ignore depletion of the fuel and put

$$
\frac{\mathrm{d} \mathscr{M}_{1}}{\mathrm{~d} t}=0 \text {. }
$$


This is a realistic procedure since the characteristic time for heat transfer is considerably shorter than that for mass changes.

3.2. Non-dimensionalisation Our aim in non-dimensionalising (6)-(10) is to maximise ease-of-understanding of our results and to retain parameters of interest as distinct continuation parameters. To achieve this we non-dimensionalise the temperature scales with respect to a reference temperature, rather than activation energy; taking $T_{\text {ref }}=298(\mathrm{~K})$.

Our primary bifurcation parameter is the concentration of oxygen in the inflow gases $\left(\mathscr{O}_{2, a}\right)$. Consequently we do not non-dimensionalise the oxygen concentration against its inflow concentration. Instead we choose as a reference concentration the concentration of oxygen in air under standard conditions $\left(\mathscr{O}_{2, r}\right)$.

We normalise the mass of the sample to be unity and the concentration of gaseous fuel so that its maximum value, in a transition calculation, is also unity, corresponding to full pyrolysis of the test sample under conditions where the gas-phase reactions are inactive.

A feature of our non-dimensionalisation is that each of the dimensional variables that we are interested in (such as characteristic temperature, pyrolysis activation energy, exothermicity and endothermicity of the oxidation reaction) appears in only one non-dimensional variable $\left(T_{c}^{*}, E_{s}^{*}, Q_{g}^{*}\right.$ and $\left.Q_{i}^{*}\right)$.

3.3. Non-dimensionalised equations In non-dimensionalising (6)-(10) we introduce non-dimensionalised temperatures $\left(T_{i}^{*}\right)$, with non-dimensionalised concentrations $\left(\mathscr{M}_{1}^{*}, \mathscr{M}_{2}^{*}, \mathscr{O}_{2}^{*}\right)$, and a non-dimensionalised time-scale $\left(t^{*}\right)$. We then obtain

$$
\begin{aligned}
& \frac{\mathrm{d} T_{g}^{*}}{\mathrm{~d} t^{*}} \frac{1}{c_{i}^{*}}=Q_{g}^{*} A_{g}^{*} \mathscr{O}_{2, r}^{*} e^{-E_{g}^{*} / T_{g}^{*}} \mathscr{M}_{2}^{*} \mathscr{O}_{2}^{*}-Q_{i}^{*} A_{i}^{*} e^{-E_{i}^{*} / T_{g}^{*}} \mathscr{M}_{2}^{*} \\
& -S^{*} \chi^{*}\left(T_{g}^{*}-T_{0}^{*}\right)-\left(T_{g}^{*}-T_{s}^{*}\right) \text {, } \\
& \frac{\mathrm{d} T_{s}^{*}}{\mathrm{~d} t^{*}}=-Q_{s}^{*} \frac{A_{s}^{*} E_{s}^{*}}{T_{c}^{* 2}} e^{E_{s}^{*}\left(T_{s}^{*}-T_{c}^{*}\right) / T_{c}^{*} T_{s}^{*}} \mathscr{M}_{1}^{*}+\left(T_{g}^{*}-T_{s}^{*}\right), \\
& \frac{\mathrm{d} \mathscr{M}_{2}^{*}}{\mathrm{~d} t^{*}}=\frac{A_{s}^{*} E_{s}^{*}}{T_{c}^{* 2}} e^{E_{i}^{*}\left(T_{s}^{*}-\tau_{c}^{*}\right) / T_{c}^{*} T_{s}^{*}} \mathscr{M}_{1}^{*}-A_{g}^{*} \mathscr{O}_{2, r}^{*} e^{-E_{\xi}^{*} / T_{s}^{*}} \mathscr{M}_{2}^{*} \mathscr{O}_{2}^{*} \\
& -A_{i}^{*} e^{-E_{i}^{*} / T_{k}^{*}} \mathscr{M}_{2}^{*}-V^{*} L^{*} \mathscr{M}_{2}^{*} \text {, } \\
& \frac{\mathrm{d} \mathscr{O}_{2}^{*}}{\mathrm{~d} t^{*}} \frac{1}{V^{*}}=-A_{g}^{*} e^{-E_{\xi}^{*} / T_{\xi}^{*}} \mathscr{M}_{2}^{*} \mathscr{O}_{2}^{*}+A_{3}^{*} e^{-E_{\mathbf{3}} / T_{\xi}^{*}}\left\{\mathscr{O}_{2, a}^{*}-\mathscr{O}_{2}^{*}\right\}, \\
& \frac{\mathrm{d} \mathscr{M}_{1}^{*}}{\mathrm{~d} t^{*}}=-A_{s}^{*} e^{-E_{s}^{*} / T_{s}^{*}} \mathscr{M}_{1}^{*} \text {, } \\
& T_{g}^{*}(0)=T_{s}^{*}(0)=\mathscr{M}_{1}^{*}(0)=\mathscr{O}_{2}^{*}(0)=1, \quad \mathscr{M}_{2}^{*}(0)=0 .
\end{aligned}
$$

The terms appearing in (15)-(20) are defined in a related paper [7]. 
In writing the non-dimensionalised equations we have explicitly included the definition of the pyrolysis pre-exponential factor in terms of a characteristic temperature. We investigate the steady-state solutions of (15)-(18), ignoring consumption of the polymer.

The percentile limiting oxygen index is defined in terms of the critical nondimensionalised oxygen concentration in the inflow gases found by

$$
L O I=100 \frac{\mathscr{O}_{2, a_{c r}}^{*}}{4.773}
$$

where 4.773 is the non-dimensionalised number of moles of an ideal gas per unit volume under standard conditions $\left(41.592 \mathrm{~mol} \mathrm{~m}^{-3}\right)$.

3.4. Numerics The path following software program Auto 94 [2] was used to obtain steady-state and bifurcation diagrams for (15)-(18). In steady-state diagrams the standard representation is used: solid lines are stable steady states and dotted lines are unstable steady states. We are interested in determining under which conditions a flame can be supported over the sample. Accordingly we principally investigate the variation of non-dimensionalised gaseous temperature $\left(T_{g}^{*}\right)$ with non-dimensionalised oxygen concentration in the inflow gases $\left(\mathscr{O}_{2, a}^{*}\right)$.

We refer to limit points as (L1), (L2), etc., where (L1) is the first limit point to be reached if one traces the steady-state curve, starting at the non-flaming steady state corresponding to zero non-dimensionalised oxygen concentration in the inflow gases.

\section{Results}

Recall that there is a one-to-one relationship between our non-dimensionalised variables and their dimensionalised counterparts. Hence we refer often to oxygen concentration rather than non-dimensionalised oxygen concentration, etc.

4.1. The structure of the steady-state solutions Figure 2 (a) shows a typical steadystate diagram. The features of this include: a locus of stable steady-state solutions that are terminated at the (L1) limit-point; a locus of unstable steady-state solutions, existing between the (L1) and (L2) limit-points; and a locus of stable steady-state solutions that are generated at the (L2) limit-point. The (L1) and (L2) limit-points are also referred to as the ignition limit-point and extinction limit-point respectively.

The first set of stable solutions is characterised by a 'low' gaseous temperature, corresponding to experimental conditions where very little reaction occurs in the gas-phase, the gas-phase temperature is close to that of the reactor-gases, and no flame is present. The second set of stable solutions is characterised by a 'high' 


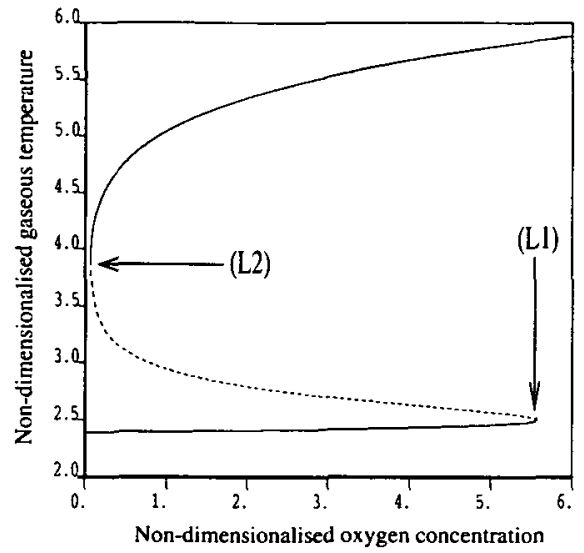

(a) Steady-state curve when characteristic temperature is $T_{c}=566.2(\mathrm{~K})$.

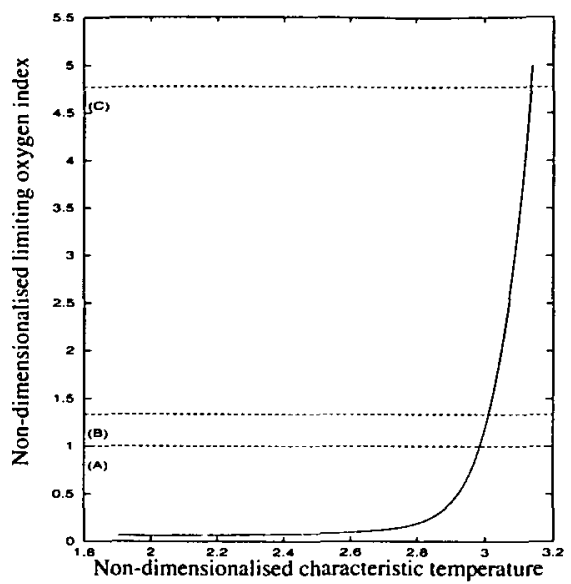

(b) Extinction limit point bifurcation diagram.

FIGURE 2. The dependence of the non-dimensionalised critical inflow concentration $\left(\mathscr{O}_{2, a_{c r}}^{*}\right)$ with characteristic temperature $\left(T_{c}^{*}\right)$ is determined by unfolding the defining limit point on the steady-state curve (L2) with the appropriate secondary bifurcation parameter. In (b) the lines $(\mathrm{A}-\mathrm{C})$ correspond to limiting oxygen indices of 20.95, representing "marginally stable" materials; 28.00 , the transition to "self-extinguishing" behaviour; and 100.00, the transition to "intrinsically non-flammable" materials, respectively. Figure (b) corresponds to a horizontal slice through Figure 4 (a) at $E_{s}^{*}=32.288$. (C2001, The Institute of Physics. Reprinted from [7].

gaseous temperature, corresponding to experiments where a stable flame burns over the sample.

When the concentration of oxygen in the inflow is less than its value at the extinction limit-point the material can not sustain a flame in the absence of a external heat-source. Therefore the limiting oxygen index is the inflow concentration at this limit point.

The purpose of our model is to investigate flame stability in the absence of an external heat source. It is assumed that the role of the heat-source used in oxygenindex experiments is to raise the temperature of the gas-phase to the flame temperature, igniting the material in the process. The heat-source is then removed and the ability of the test sample to undergo self-sustaining combustion is investigated by varying the inflow-concentration-replicating experimental procedure. Thus the ignition limitpoint is not related to ignitability of the sample in oxygen-index experiments.

The ignition limit-point defines an upper flammability limit-should the sample be contained in an atmosphere containing a greater oxygen concentration than that at the ignition limit point then it will spontaneously ignite in the absence of an external heat source.

4.2. Unfolding the limit point In Section 4.1 we discussed how a limiting oxygen index can be defined as the oxygen concentration in the inflow gases at the extinction 


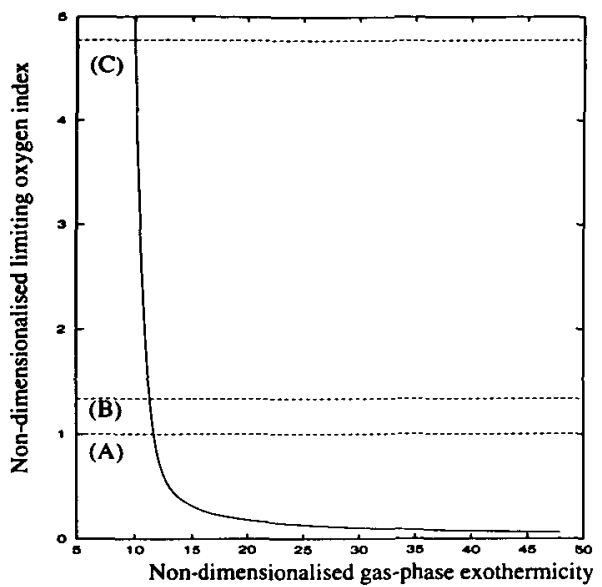

(a)

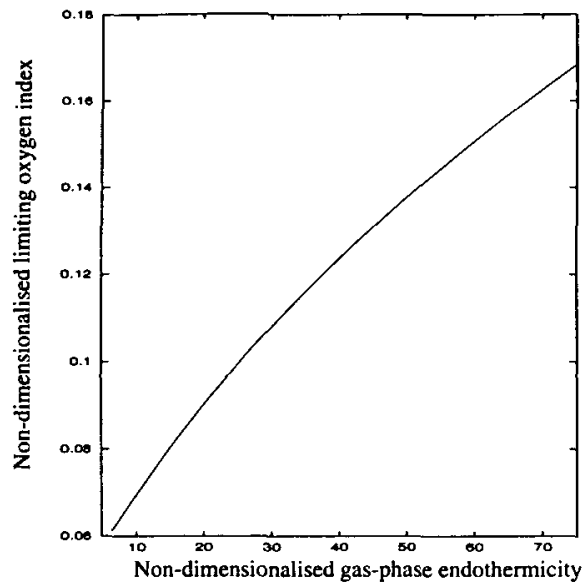

(b)

FIGURE 3. The variation of non-dimensionalised limiting oxygen index $\left(\mathscr{O}_{2, a_{c r}}^{*}\right)$ : (a) non-dimensionalised gas-phase exothermicity $\left(Q_{g}^{*}\right)$; and (b) non-dimensionalised gas-phase endothermicity $\left(Q_{i}^{*}\right)$, determined by unfolding the defining limit point on the steady-state curve, (L2) in Figure 2 (a). In (a) the lines (A-C) correspond to limiting oxygen indices of 20.95 , representing "marginally stable" materials; 28.00 , the transition to "self-extinguishing" behaviour; and 100.00 , the transition to "intrinsically non-flammable" materials, respectively.

limit point. Figure 2 (b) shows the dependence of the oxygen index upon the characteristic temperature, determined by continuation. The starting point for the continuation is the extinction limit point shown in Figure 2 (a). Lines (A) and (C) correspond to limiting oxygen indices of 20.95 and 100.0, representing materials where the flame is only just stable in either standard conditions or in an atmosphere of $100 \%$ oxygen respectively. Line (B), corresponding to a limiting oxygen index of 28 , represents the transition between "self-extinguishing" and "non self-extinguishing" polymers.

Figure 3 shows how the oxygen index depends upon gas-phase exothermicity, (a), and gas-phase endothermicity, (b). In both cases the starting point for continuation is the extinction limit point in Figure 2 (a). Figure 3 (a) shows that it is possible to make this material "marginally stable", that is, to increase its limiting oxygen index to 20.95 , by reducing the exothermicity of the gas-phase reactions by approximately two-thirds of its initial value whereas Figure 3 (b) shows that increasing the endothermicity of the 'incomplete' combustion reactions by a factor of ten does not achieve this aim. These results can be interpreted in terms of the effectiveness of fire-retarding mechanisms and hence the effectiveness of different fire-retardants, in reducing the flammability of the material represented by Figure 2 (a).

4.3. Regions of flammability and "self-extinguishing" behaviour In Figure 2 (b) the pyrolysis activation energy was held constant and the extinction limit point un- 


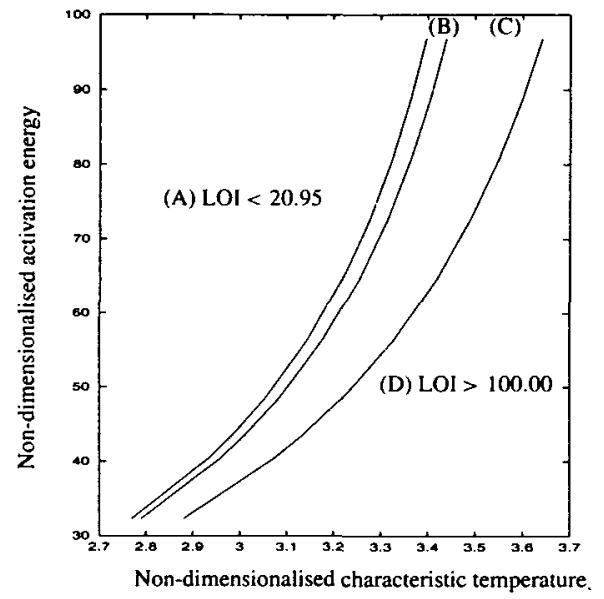

(a)

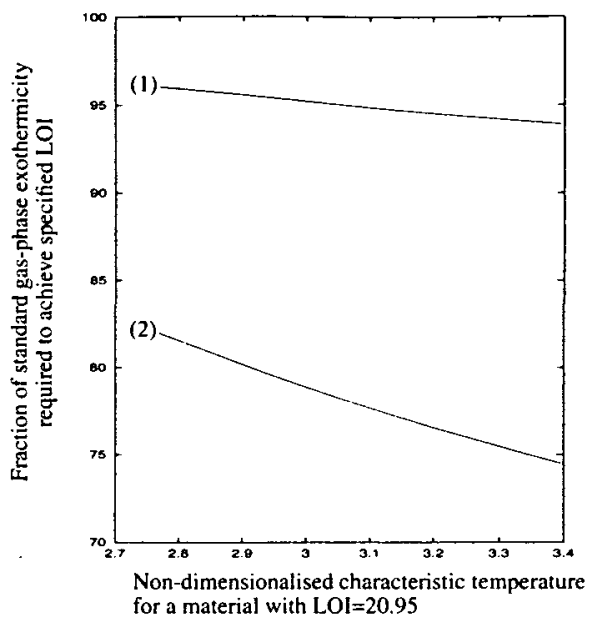

(b)

FIGURE 4. (a) The classification of materials into categories depending upon their pyrolysis kinetics: (A) LOI $<20.95$; (B) $20.95<\mathrm{LOI}<28.0$; (C) $28.0<$ LOI $<100.0$, corresponding to materials that are "self-extinguishing"; and (D) LOI $>100.0$. (b) The fractional change in gas-phase exothermicity $\left(100 \times Q_{g}^{*} / 47.939\right)$ that is required to increase the LOI of marginally stable materials: (1) LOI $=28.0$, the threshold for "self-extinguishing" materials, and (2) LOI $=100.0$, the threshold for "intrinsically non-flammable" materials.

folded with characteristic temperature. The materials represented in Figure 2 (b) are classed into four categories: (A) LOI $<20.95$; (B) $20.95<$ LOI $<28.00$; (C) $28.00<$ LOI $<100.00$, "self-extinguishing" materials; and (D) LOI $>100.00$, "intrinsically non-flammable" materials. Figure 4 (a) shows how these regions depend upon both the pyrolysis activation energy and the characteristic temperature, showing that for fixed characteristic temperature flammability increases with increasing activation energy whilst for fixed activation energy flammability decreases with increasing characteristic temperature.

Figure 3 (a) shows that, for the material represented by Figure 2 (a), the exothermicity of the gas-phase reaction must be reduced by approximately $75 \%$ to make it self-extinguishing, that is, to increase its limiting oxygen index to 28 . In fact, the oxygen index of this material is so low that, in practice, one would not seek to decrease its flammability, but would instead select a different material. For a given value of the characteristic temperature the activation energy of a marginally stable material is defined in Figure 4 (a). Figure 4 (b) shows the value of the gas-phase exothermicity, expressed as a fraction of the standard value, at which such materials become "self-extinguishing", line 1, and "intrinsically non-flammable", line 2. A reduction of approximately $6 \%$ makes a "marginally stable" material "self-extinguishing", the requirement varying only slightly with the pyrolysis kinetics. 


\section{Discussion}

Oxygen index is inversely proportional to polymer flammability and directly proportional to resistance to flame propagation. The efficiency of particular flame-retardants can therefore be investigated in terms of the change in the LOI that they induce [5]. The simplest approach to modelling fire-retardants is to investigate the behaviour of the model as an appropriate bifurcation parameter is varied. For instance, Figure 3 can be considered as modelling the action of two particular types of gas-phase active fire-retardants. Although we have interpreted Figures 2 (b) and 4 (a) in terms of materials having different pyrolysis kinetics they could be interpreted in terms of an additive that changes the pyrolysis reactions in the condensed phase.

It is convenient to divide materials into four classes depending upon their oxygen index, see Figure 4 (a). The transition from region (C) to (D) is characterised by a so-called boundary bifurcation. These occur when a bifurcation point passes from a region that is physically acceptable to one that is not, in this case from LOI $<100$ to LOI $>100$. The influence of such 'bifurcations' in determining system behaviour in chemical kinetic models was first discussed by Gray and Roberts, [3, 4].

The dependence of oxygen index upon model parameters was investigated by Búcsi and Rychlý [1] using direct integration, requiring many runs. The steady-state formulation provides a natural way in which to systematically investigate their effect. We are currently investigating the effectiveness of various fire-retardancy mechanisms, concentrating on the requirements to render a "marginally stable" material "selfextinguishing", see Figure 4 (b).

\section{Conclusions}

A dynamical systems model of the limiting oxygen-index test has been derived, expanding upon work by Rychlý and his co-workers [1,8-10]. Using a steady-state formulation we have identified the oxygen index of a material with the extinction limit point in its steady-state diagram and illustrated how the action of fire-retardants can be modelled by unfolding this bifurcation with an appropriate continuation parameter.

\section{Acknowledgements}

This work was carried out whilst MIN was supported by a fellowship from the Royal Society of London to work in the Department of Mathematics and Statistics at the University of Canterbury (New Zealand). Support from the Australian Defence Force Academy is also acknowledged. 


\section{A. Parameter values}

Unless otherwise specified we take the following typical parameter values:

$\begin{array}{lll}A_{g}=e^{12.6} \mathrm{~m}^{3} \mathrm{~mol}^{-1} \mathrm{~s}^{-1}, & A_{i}=e^{27.8} \mathrm{~s}^{-1}, & A_{3}=e^{15.3} \mathrm{~s}^{-1}, \\ E_{g}=88 \times 10^{3} \mathrm{~J} \mathrm{~mol}^{-1}, & E_{i}=243 \times 10^{3} \mathrm{~J} \mathrm{~mol}^{-1}, & E_{s}=108 \times 10^{3} \mathrm{~J} \mathrm{~mol}^{-1}, \\ E_{3}=138 \times 10^{3} \mathrm{~J} \mathrm{~mol}^{-1}, & \mathscr{H}=\frac{1}{60} \mathrm{~K} \mathrm{~s}^{-1}, & O_{2 . r}=8.714 \mathrm{~mol} \mathrm{~m}^{3}, \\ Q_{g}=3000 \times 10^{3} \mathrm{~J} \mathrm{~mol}^{-1}, & Q_{i}=400 \times 10^{3} \mathrm{~J} \mathrm{~mol}^{-1}, & Q_{s}=750 \times 10^{3} \mathrm{~J} \mathrm{~mol}^{-1}, \\ S_{g . o}=5.0 \times 10^{-2} \mathrm{~m}^{2}, & S_{g . s}=10^{-2} \mathrm{~m}^{2}, & T_{0}=723 \mathrm{~K}, \\ T_{c}=580 \mathrm{~K}, & V_{g}=10^{-3} \mathrm{~m}^{3}, & V_{s}=10^{-5} \mathrm{~m}^{3}, \\ c_{p_{s}}=1300 \mathrm{~J} \mathrm{~kg}^{-1} \mathrm{~K}^{-1}, & c_{p_{s}}=1300 \mathrm{~J} \mathrm{~kg}^{-1} \mathrm{~K}^{-1}, & n=10 \mathrm{~mol} \mathrm{~kg}^{-1}, \\ \rho_{g}=1.0 \mathrm{~kg} \mathrm{~m}^{-3}, & \rho_{s}=2000 \times 10^{3} \mathrm{~kg} \mathrm{~m}^{-3}, & \chi_{g . o}=74 \mathrm{~J} \mathrm{~s}^{-1} \mathrm{~m}^{-2} \mathrm{~K}^{-1}, \\ \chi_{g . s}=\chi_{s . a}=37 \mathrm{~J} \mathrm{~s}^{-1} \mathrm{~m}^{-2} \mathrm{~K}^{-1} . & \end{array}$

The appropriate values for physical constants are: $R=8.31441 \mathrm{~J} \mathrm{~K} \mathrm{~mol}^{-1}$.

\section{References}

[1] A. Búcsi and J. Rychlý, "A theoretical approach to understanding the connection between ignitability and flammability parameters of organic polymers", Polymer Degradation and Stability 38 (1992) 33-40.

[2] E. Doedel, X. Wang and T. Fairgrieve, "AUTO 94 Software for Continuation and Bifurcation Problems in Ordinary Differential Equations", Applied Mathematics Report California Institute of Technology Pasadena. California 91125, November 1994.

[3] B. F. Gray and M. J. Roberts, "Analysis of chemical kinetic systems over the entire parameter space I. The Sal'nikov thermokinetic oscillator", Proc. Roy. Soc. A 416 (1988) 391-402.

[4] B. F. Gray and M. J. Roberts, "Analysis of chemical kinetic systems over the entire parameter space II. Isothermal oscillators", Proc. Roy. Soc. A 416 (1988) 403-424.

[5] A. R. Horrocks, M. Tunc and D. Price. "The burning behaviour of textiles and its assessment by oxygen-index methods", Textile Progress 18 (1989) 1-205.

[6] M. I. Nelson, "Ignition mechanisms of thermally thin thermoplastics in the cone calorimeter", Proc. Roy. Soc. A 454 (1998) 789-814.

[7] M. I. Nelson, "A dynamical systems model of the limiting oxygen index test. II Retardancy due to char-formation and addition of inert fillers", Combustion Theory and Modelling 5 (2001) 59-83.

[8] J. Rychlý and L. Costa, "Modelling of polymer ignition and burning adopted for cone calorimeter measurements: the correlation between the rate of heat release and oxygen index", Fire and Materials 19 (1995) 215-220.

[9] J. Rychlý and L. Rychlá, "Effect of flame retardants on polyolefines", Fire and Materials 10 (1986) 7-10.

[10] J. Rychlý and L. Rychlá, "Modelling of heat-release rate-time curves from cone calorimeter for burning of polymers with intumescence additives", Polymer Degradation and Stability 54 (1996) 249-254. 